\title{
Wind Tunnel Data Fusion and Immersive Visualization: A Case Study
}

\author{
Kurt Severance \\ NASA Langley Research Center \\ k.severance@larc.nasa.gov
}

\author{
Paul Brewster \\ NASA Langley Research Center \\ p.f.brewster@larc.nasa.gov
}

\author{
Barry Lazos \\ NASA Langley Research Center \\ b.s.lazos@larc.nasa.gov
}

\author{
Daniel Keefe \\ Brown University \\ dfk@cs.brown.edu
}

\begin{abstract}
This case study describes the process of fusing the data from several wind tunnel experiments into a single coherent visualization. Each experiment was conducted independently and was designed to explore different flow features around airplane landing gear. In the past, it would have been very difficult to correlate results from the different experiments. However, with a single 3-D visualization representing the fusion of the three experiments, significant insight into the composite flowfield was observed that would have been extremely difficult to obtain by studying its component parts. The results are even more compelling when viewed in an immersive environment.
\end{abstract}

CR Categories: I.3.7 [Computer Graphics]: Three-Dimensional Graphics and Realism - color, shading, and texture; I.3.8 [Computer Graphics]: Applications.

Keywords: Data fusion, Photogrammetry, Line Integral Convolution, Reconstruction, Oil flow, Particle Image Velocimetry, Wind tunnel, Landing gear, Texture mapping, Image-based rendering, VRML.

\section{INTRODUCTION}

Experimental wind tunnel data come in various forms and with varying quality. Unlike computational fluid dynamics (CFD), where the values at each grid point are calculated, wind tunnel experiments use various sensors to try to directly and indirectly measure values in certain areas. Measuring the complete flow around an object, similar to a CFD solution, is often impractical, if not impossible. The data could be quantitative information like pressure on the surface, or qualitative information, like pictures or images of flow patterns. It can also be difficult to correlate data taken from different measurements of the same object, even at the same flow conditions. This case study gives an example of fusing three different kinds of image and point measurements together into a single 3-D flow visualization that can be displayed in an immersive environment.

Aircraft noise has been one of the key difficulties in building an efficient civil air network. Because of the noise, airports are often built farther from urban populations than is practical. Next to the engine, the landing gear during final approach can be one of the largest generators of noise. Noise is created by pressure fluctuations in the fluid flow around the gear. Three experiments were conducted to analyze the mean flow of air around the landing gear of a Boeing 757. This information may be used to validate computational results and highlight areas where more detailed time dependent measurements are important. The experiments are explained in detail in [8], but a brief explanation of pertinent parts of the experiment follow.

\section{WIND TUNNEL EXPERIMENTS}

The experiments were conducted in the Basic Aerodynamics Research Tunnel (BART) [9] at the NASA Langley Research Center. The model was a generic 4-wheel landing gear configuration with components scaled to $31 \%$ of the main landing gear of a Boeing 757 (Figure 1). The model was simplified by eliminating detailed components such as tubing, wheel covers, braking mechanisms, bolt heads, etc. Three experiments were performed on this model:

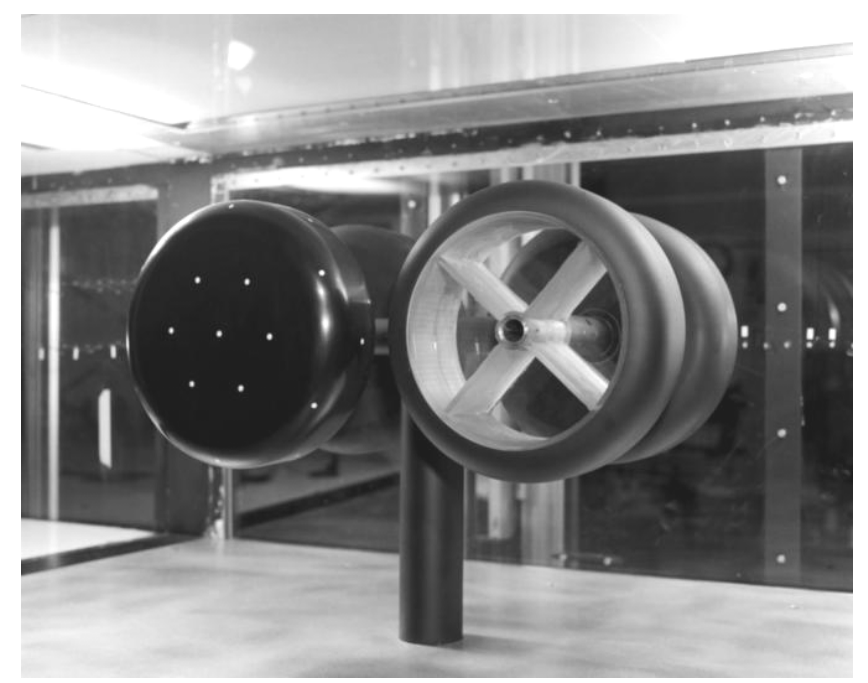

Figure 1. Inverted landing gear model in the wind tunnel. Flow direction is from left to right.

1. A mixture of oil (kerosene) and titanium dioxide powder was applied to a single wheel. The tunnel was rapidly brought to test speed, and shear lines formed in the mixture as air flowed over the wheel. Photographs of the established flow patterns were then obtained from multiple angles using a digital camera. A sample photograph of the front wheel is shown in Figure 2. This same wheel was cleaned and mounted on the rear axle, the mixture was reapplied, and the wind tunnel was rapidly brought to the same flow conditions. After the mixture was dry, similar images were taken of the established shear stress patterns on the rear wheel.

2. Surface pressures were measured using a line of pressure taps along the wheel periphery from the inside center (near the axle) to the outside center. Pressure readings over most of the surface of the wheel were then obtained by rotating the wheel about its axle in 2-degree increments in a stepwise fashion. At each step, 30,000 data samples were taken per sensor. These samples were averaged together to compute the mean pressure at each point.

3. Digital Particle Image Velocimetry (DPIV) [7] was used to determine the mean velocities in a vertical plane bisecting the inline wheels. This procedure involved illuminating flow particles with a laser light sheet and recording their 
instantaneous positions using two photographs taken microseconds apart. One hundred image pairs were used to determine the mean velocities at 160 locations that formed the data plane (Figure 4).

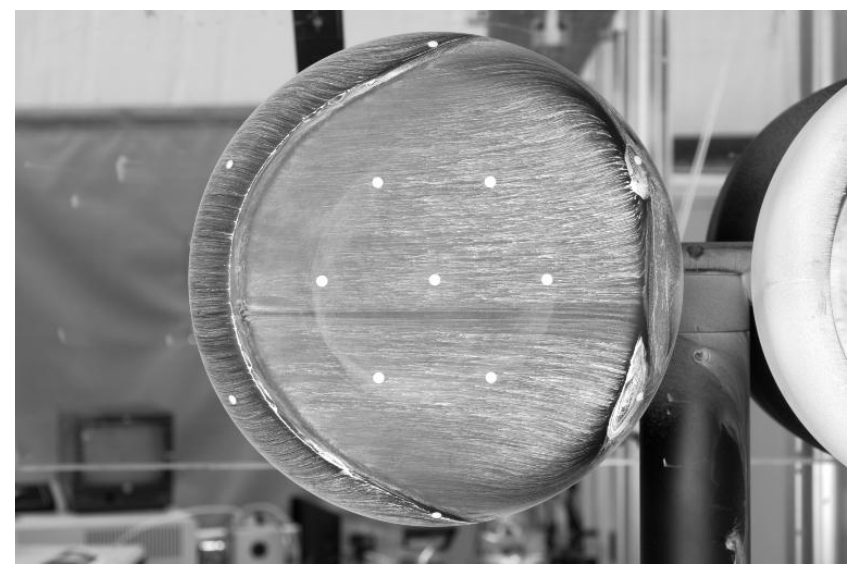

Figure 2. Photograph of the oil flow on the outside of the wheel.

\section{VISUALIZATION METHODS}

The geometry of the landing gear model was well known before the experiments. A PLOT3D structured grid of 50x181, or 9050 , points was constructed based upon the as-built measurements of the wheel. The points on the grid coincided with the pressure tap measurement locations so that surface pressures could be easily visualized. Fiducial marks were also placed at regularly spaced locations around the surface of the wheel. Their coordinates were measured to later facilitate the calibration of the digital camera.

\subsection{Oil Flow}

Once the oil flow pattern had dried on a wheel after a tunnel test, a digital camera was used to capture eight high-resolution images (3060x2036 pixels). The first image was obtained while the model was still in the tunnel (Figure 2). Then the wheel was removed and oriented horizontally by inserting the axle into a temporary mount. Six more images were then captured around the periphery of the wheel, at locations aligned with fiducial points on the model. A final image was captured from the side of the wheel containing the axle. A schematic showing the relative placement of seven digital images is shown in Figure 3.

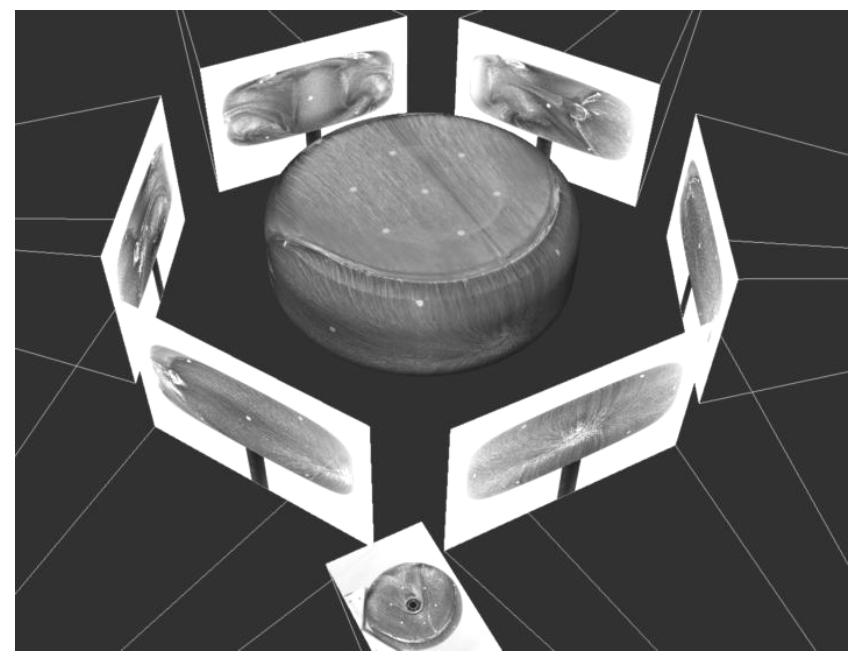

Figure 3. Relative placement of camera images around the wheel.
Given eight oil flow images of one wheel, our goal was to combine these photographs into a single 3-D visualization. We followed a procedure very similar to the photogrammetric reconstruction described in $[10,11]$ which created $3-D$ oil flow and light sheet visualizations from $2-\mathrm{D}$ video images. In fact, the camera calibration and resection procedure is the same, and is shown schematically in Figure 5. This procedure is based upon the collinearity condition in which the object point, image point, and camera lens center all lie on a straight line. Well-placed and accurately measured fiducial marks are a key input into the process. Further details on this procedure can be found in [2, 5]. The result of the camera calibration and resection process is the $\mathrm{X}, \mathrm{Y}, \mathrm{Z}$ position and $\Omega, \Phi, \mathrm{K}$ orientation angles of the camera. This procedure was performed on each of the eight camera shots.

Given the camera parameters and a known geometry, the procedure in [11] can be used to perform a forward projection from a single camera onto a 3-D surface. However, when working with multiple cameras, we found it more effective to implement a backward projection scheme. This approach is similar to the image-based rendering techniques in [4] and the multiple camera mapping technique in [6]. The algorithm examines each quadrilateral of the known surface geometry (described as a PLOT3D structured grid) and determines which camera had the best view of that facet, based upon the surface normal (Figure 6). Next, the 3-D vertices of that quadrilateral are back-projected toward the selected camera to determine the corresponding 2-D image texture coordinates (Figure 7). The pixels from this region of the camera image are then applied to the quadrilateral using standard texture mapping. This procedure is carried out for every facet of the geometry, until the entire object is texture mapped. (Currently, this algorithm uses only the surface normal to determine the visibility of a facet, thus only convex surfaces are supported at this time.) The output is expressed as a single platform-independent VRML 1.0 file. The result is a single 3 -D visualization with very high-resolution textures that convey the complex oil flow patterns around the entire model. It can be viewed interactively on any platform with adequate texture mapping hardware.

\subsection{Surface Pressures}

Since the vertices of the PLOT3D grid describing the geometry of the wheel were identical to the pressure tap locations, adding pressure information to the oil flow visualization was straightforward. VRML 1.0 allows both texture and color information for each vertex, so we simply added the appropriate color from a rainbow color map to represent the pressure at each vertex. The result of combining scalar pressures represented by color with the grey-scale oil flow texture is shown in Color Plate 1. Note the correspondence of the oil flow features with both low (blue) and high (white) pressure regions. The combination of these two surface data sets into a single $3-D$ visualization made identification of the surface topology features far easier and more accurate than the manual comparison of numerous experimental 2-D images and pressure measurements.

\subsection{Digital Particle Image Velocimetry}

The DPIV process resulted in a steady 2-D Cartesian vector data set. Because the plane was measured in piecewise fashion, the data was divided into 160 cells of $60 \times 60$ data points each, with 4 points overlapping neighboring cells (Figure 4). A Gaussian filter was applied to smooth the edges between cells and combine the data into one Cartesian 1236x676 data set. Vectors with a value of 
zero were placed in the parts of the data set corresponding to the interior of the wheels.

The Line Integral Convolution (LIC) [1] technique was applied to visualize the data in a manner visually similar to the flow of oil on the wheels. Color was used to visualize the magnitude of the velocity at each point. This process resulted in a single color image showing the flow of air across the plane (Color Plate 2). A vortex is apparent between the wheels, and a saddle point and slower moving air appear behind the wheels.

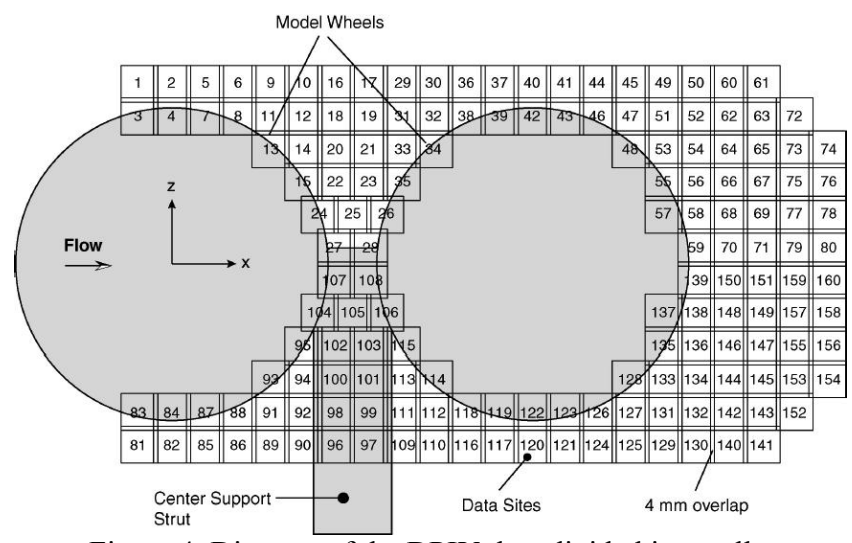

Figure 4. Diagram of the DPIV data divided into cells. Flow direction is from left to right.

\subsection{Immersive Visualization}

The on-surface oil flow and pressure visualizations for the front and rear wheels were combined into a single VRML 1.0 file. Flow symmetry was assumed to produce visualizations of all four wheels. The 2-D LIC image (from Color Plate 2) representing the off-surface velocity field data was then texture-mapped onto the appropriate 3-D plane between the wheels. Color was removed and transparency was added to the LIC image to facilitate comparison with on-surface flow features. A few well-placed streamlines were also included to highlight certain flow features in the data set. The final scene (Color Plate 3) was presented on an ImmersaDesk and in a CAVE [3], where the researcher could navigate around a compelling visualization of the experiments and obtain a fully 3-D view from any point. This immersive environment was particularly effective for examining flow behavior in very tight locations, such as in-between wheels. It was very straightforward in this environment for the researcher to correlate the off-surface flow features (e.g., vortices and stagnation points) with on-surface flow signatures (e.g., convergence and separation zones). The point of view within the interior of a (hollow) wheel was also effective within the CAVE. This vantage point provided the user with an overview of the flow patterns around that wheel, any part of which could be observed simply by turning their head. In the future, more user-interaction will be added to this environment, to select from options such as animated LIC, multiple flow conditions, and scalar color control.

\section{CONCLUSIONS}

This case study described the process by which data from several very different wind tunnel experiments can be combined into a single 3-D scene by applying appropriate visualization techniques. The result is an accurate high-resolution reconstruction of the composite flowfield that allows the researcher to visualize and correlate the data from multiple experiments all at once. This capability provides valuable insight into the flowfield that would be nearly impossible to obtain by studying its component parts. The alternative, a manual comparison of more than a dozen 2-D oilflow photographs with numerous pressure and velocity measurements, would be very impractical. The $3-\mathrm{D}$ visualization also serves as a useful record of the experiment that can be shared with others on the Web via VRML. It also became apparent that the best quality visualizations were obtained when the experiments are designed from the beginning with visualization in mind. Specifically, well placed and accurately measured fiducial marks, a high quality digital camera, sufficient lighting, and proper camera settings are necessary to produce high-quality results.

\section{ACKNOWLEDGEMENTS}

The visualization work was performed in the Data Visualization and Analysis Lab at NASA Langley Research Center. Special thanks to Patricia Kerr for geometry preparation, David Wolverton for camera calibration, and Chris Sandridge for providing the ImmersaDesk and CAVE. Thanks also to Jay Lambiotte, Kathy Stacy, and Ronnie Gillian for their advice and support.

\section{REFERENCES}

[1] B. Cabral, and L. Leedom. Imaging Vector Field Using Line Integral Convolution. In Computer Graphics (SIGGRAPH 93 Proceedings), pg 263-270.

[2] CRAMPA-Close Range Multistation Photogrammetric Adjustment Reference Guide. Version 1.1, Geometric Software (Coburg, Austral.), May 1993.

[3] C. Cruz-Neira, D. Sandin, and T. DeFanti. Surround-Screen Projection-Based Virtual Reality: The Design and Implementation of the CAVE. Computer Graphics (SIGGRAPH 93 Proceedings).

[4] P. Debevec, S Gortler, L. McMillan, R. Szeliski, and C. Bregler. Image-Based Modeling and Rendering. SIGGRAPH 98 Course Notes for Course 15, July 1998.

[5] H. Karara. Non-Topographic Photogrammetry. Second ed. American Soc. For Photogrammetry and Remote Sensing, 1989.

[6] L. Keely and S. Uselton. Development of a Multi-Source Visualization Prototype. IEEE Visualization '98. IEEE, October 1998.

[7] C. Landreth and R. Adrian. Measurement and Refinement of Velocity Data using High Image Density Analysis in Particle Image Velocimetry. Applications of Laser Anemometry to Fluid Mechanics, $4^{\text {th }}$ Internation Symposium. SpringerVerlag. July 1988.

[8] B. Lazos. Mean Flow Features Around The Inline Wheels Of a 4-Wheel Landing Gear. To be published in AIAA Journal, 2001.

[9] W. Sellers III, and S. Kjelgaard. The Basic Aerodynamics Research Tunnel-A facility Dedicated to Code Validation. AIAA-88-1997, May 1988.

[10] K. Stacy, K. Severance, and B. Childers. Computer-Aided Light Sheet Flow Visualization. AIAA-93-2915, July 1993.

[11] K. Stacy, K. Severance, and B. Childers. Computer-Aided Light Sheet Flow Visualization Using Photogrammetry. NASA TP-3416, July 1994. 


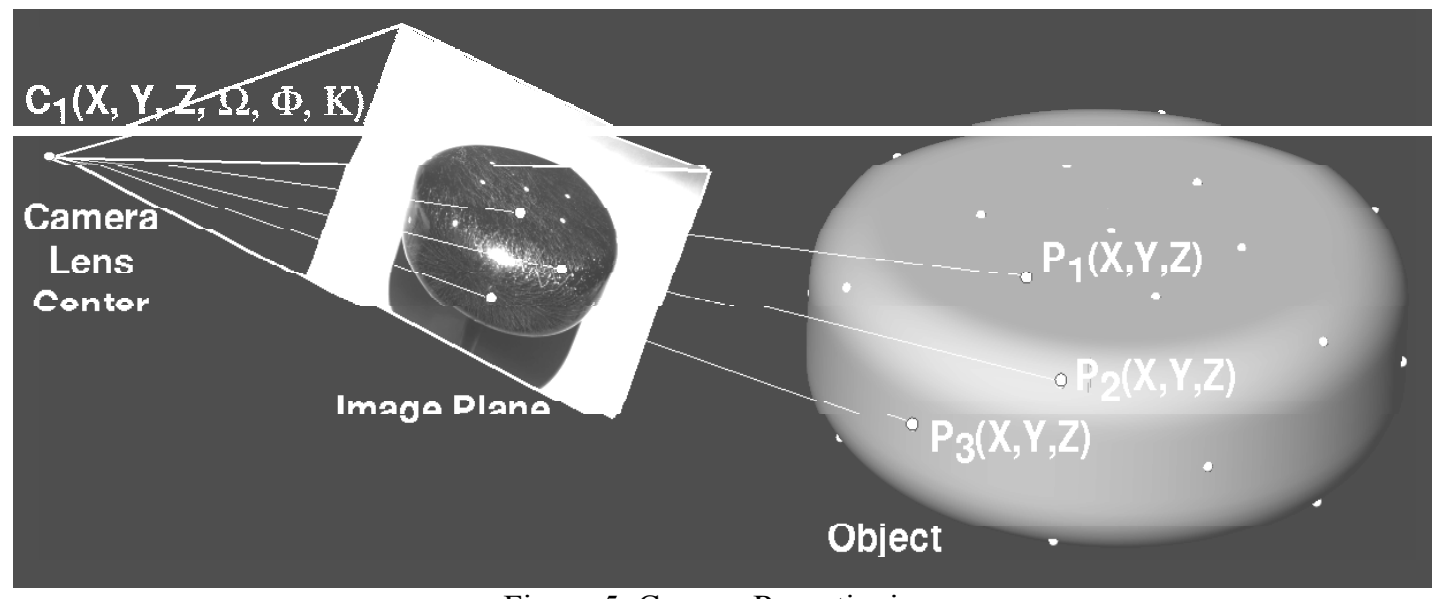

Figure 5. Camera Resectioning.

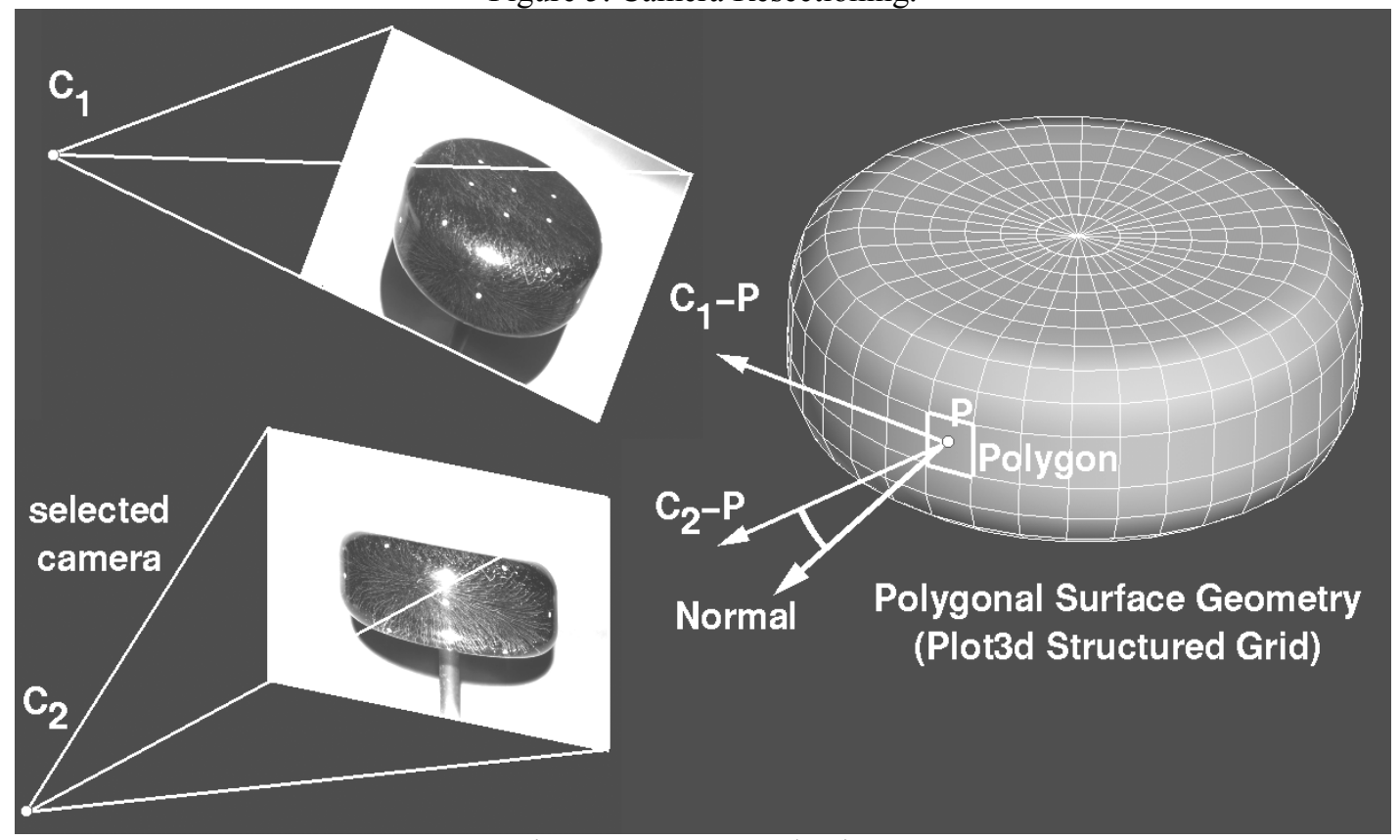

Figure 6. Camera Selection.

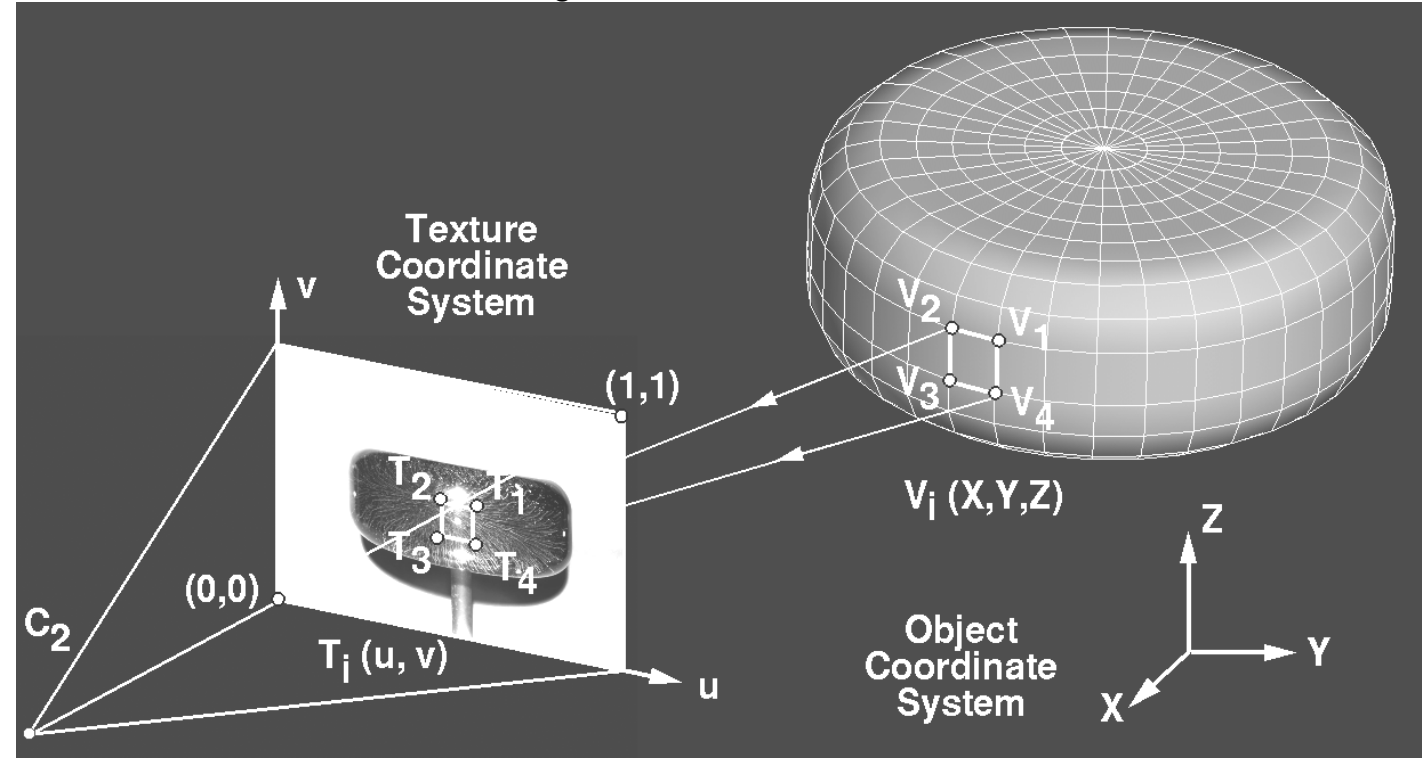

Figure 7. Texture Mapping 


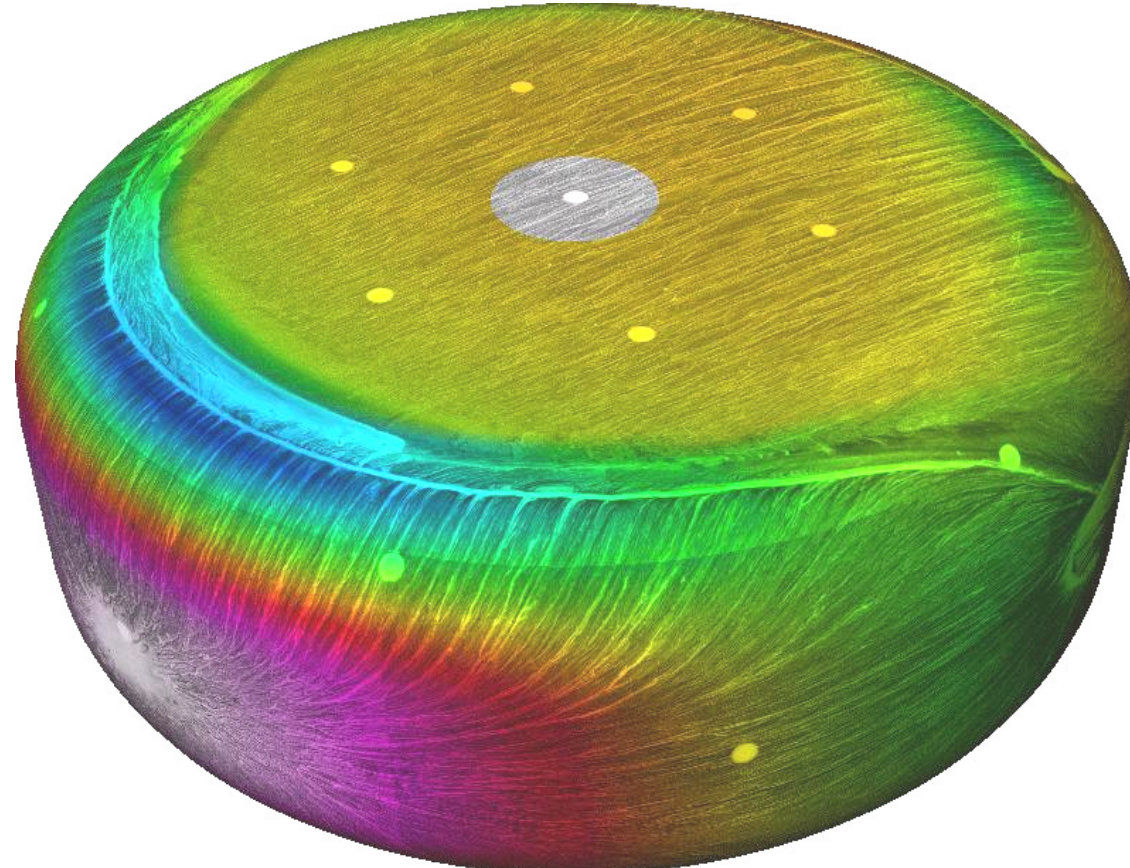

Color Plate 1. Texture mapped oil flow images colored by pressure on the front wheel. Flow direction is from lower left to upper right.

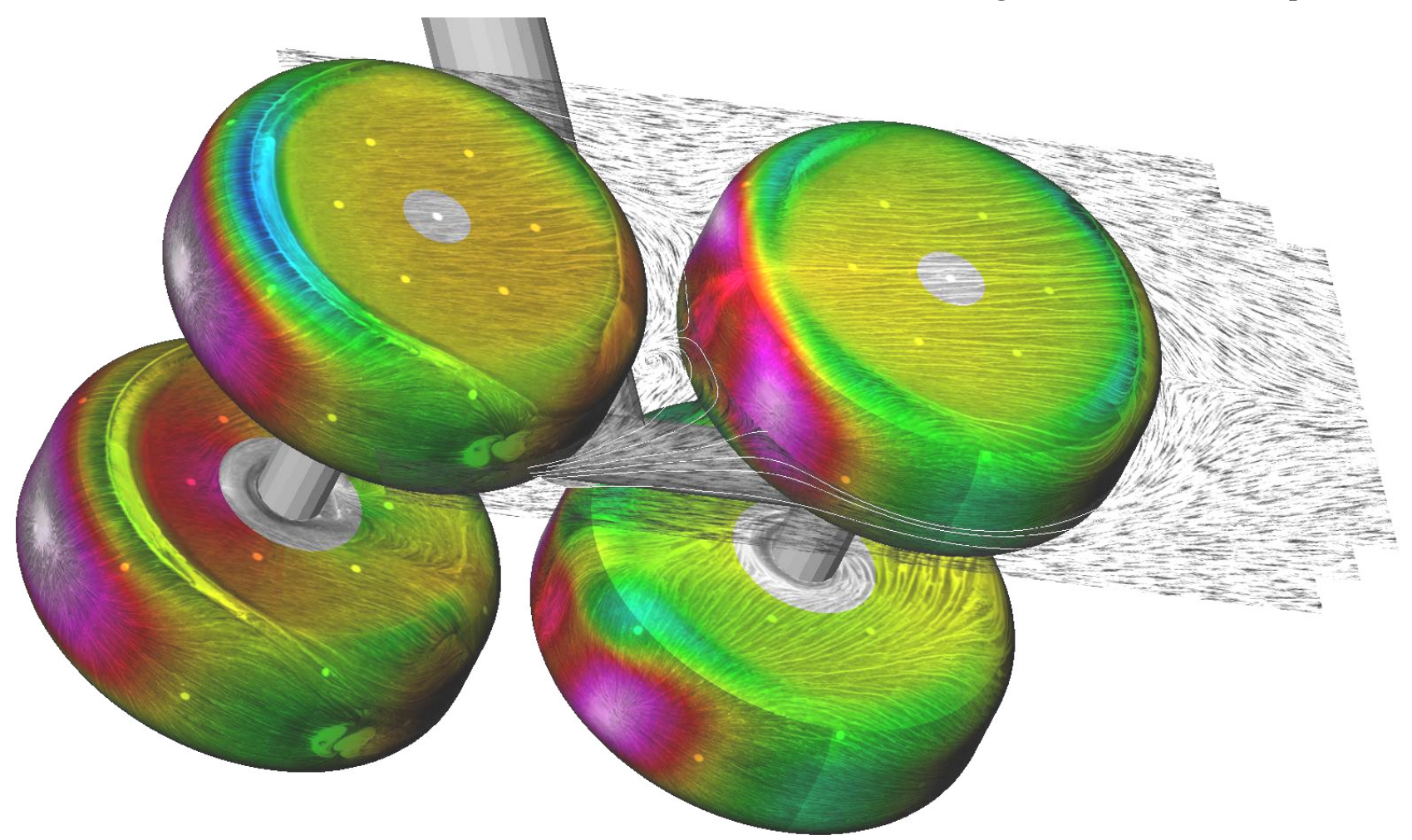

Color Plate 3. Final data fusion of oil flow images, surface pressures, and velocity measurements for the landing gear configuration. Flow direction is from left to right. 